\title{
Efficacy profile of liposome bupivacaine, a novel formulation of bupivacaine for postsurgical analgesia
}

REVIEW

This article was published in the following Dove Press journal: Journal of Pain Research

30 April 2012

Number of times this article has been viewed

Sergio D Bergese'

Sonia Ramamoorthy ${ }^{2}$

Gary Patou ${ }^{3}$

Kenneth Bramlett ${ }^{4}$

Stephen R Gorfine ${ }^{5}$

Keith A Candiotti ${ }^{6}$

'Department of Anesthesiology and Neurological Surgery, Ohio State University, Columbus, OH, USA; ${ }^{2}$ Department of Surgery, University of California at San Diego, La Jolla, CA, USA; ${ }^{3}$ Pacira Pharmaceuticals, Inc., Parsippany, NJ, USA; ${ }^{4}$ Private Practice, Alabama Orthopaedic Institute, Birmingham, AL, USA; ${ }^{5}$ Department of Surgery, The Mount Sinai Medical Center, New York, NY, USA; ${ }^{6}$ Department of Anesthesiology, University of Miami, Miami, FL, USA

Correspondence: Sergio D Bergese, MD The Ohio State University Medical Center, Department of Anesthesiology and Neurological Surgery,

$410 \mathrm{~W}$ 10th Avenue,

Doan Hall N4I I, Columbus,

$\mathrm{OH} 43210$, USA

$\mathrm{Tel}+\mathrm{I} 6142939027$

Fax +l 6142938153

Email sergio.bergese@osumc.edu
Background: Liposome bupivacaine is a novel formulation of the local anesthetic bupivacaine, designed to provide prolonged postsurgical analgesia. This analysis examined pooled efficacy data as reflected in cumulative pain scores from 10 randomized, double-blind liposome bupivacaine clinical studies in which the study drug was administered via local wound infiltration.

Methods: A total of 823 patients were exposed to liposome bupivacaine in 10 local wound infiltration studies at doses ranging from $66 \mathrm{mg}$ to $532 \mathrm{mg}$ in five surgical settings; 446 patients received bupivacaine $\mathrm{HCl}$ (dose: $75-200 \mathrm{mg}$ ) and 190 received placebo. Efficacy measures were assessed through 72 hours after surgery.

Results: Overall, $45 \%$ of patients were male and $19 \%$ were $\geq 65$ years of age. In the analysis of cumulative pain intensity scores through 72 hours, liposome bupivacaine was associated with lower pain scores than the comparator in 16 of 19 treatment arms assessed, achieving statistically significant differences compared with bupivacaine $\mathrm{HCl}(P<0.05)$ in five of 17 treatment arms. These results were supported by results of other efficacy measures, including time to first use of opioid rescue medication, proportion of patients avoiding opioid rescue medication, total postsurgical consumption of opioid rescue medication, and patient/care provider satisfaction with postoperative analgesia. Local infiltration of liposome bupivacaine resulted in significant systemic plasma levels of bupivacaine, which could persist for 96 hours; systemic plasma levels of bupivacaine following administration of liposome bupivacaine were not correlated with local efficacy. Liposome bupivacaine and bupivacaine $\mathrm{HCl}$ were generally well tolerated.

Conclusion: Based on this integrated analysis of multiple efficacy measures, liposome bupivacaine appears to be a potentially useful therapeutic option for prolonged reduction of postsurgical pain in soft tissue and orthopedic surgeries.

Keywords: pain, postsurgical; wound infiltration; local anesthetic; analgesic

\section{Introduction}

Most patients experience moderate to extreme pain after surgery, ${ }^{1-3}$ and effective postsurgical pain management is a key factor affecting patient recovery. ${ }^{4,5}$ Multimodal analgesia techniques involving analgesics, such as local anesthetics, oral or parenteral nonsteroidal anti-inflammatory medications, and oral or parenteral opioids, are recommended as the most safe and effective approach to postsurgical pain control. ${ }^{4}$

Local anesthetics administered during surgery are frequently used as part of multimodal analgesic regimens; however, the duration of analgesia with these agents is short ( $<12$ hours). ${ }^{6-9}$ Bupivacaine has a long history of use in the surgical setting, and the efficacy of bupivacaine $\mathrm{HCl}$ administered perioperatively via wound infiltration for acute postsurgical pain is well established..$^{6-8,10-12}$ A novel formulation of bupivacaine, 
ie, liposome bupivacaine (Pacira Pharmaceuticals, Inc., Parsippany, NJ), has been developed to address the need for longer-acting local anesthetics that can be administered as a single dose. This article provides an overview of the efficacy profile of liposome bupivacaine based on Phase II and Phase III data from 10 randomized, double-blind, controlled, singledose wound infiltration studies in patients undergoing hernia repair, total knee arthroplasty, hemorrhoidectomy, breast augmentation, or bunionectomy.

\section{Materials and methods}

All 10 studies were performed in compliance with the Declaration of Helsinki and its amendments and Good Clinical Practice. ${ }^{13,14}$ Prior to enrolling patients, each study site obtained the approval of its institutional review board and/or ethics committee. Written informed consent was obtained from all patients or a legal surrogate, or the requirement for written informed consent was waived by the ethics committee.

Participants in all studies were adults $\geq 18$ years of age who were scheduled to undergo the specified surgical procedure in each study. Patients were excluded if they had a concurrent painful physical condition or concurrent surgery that could have required analgesic treatment in the postsurgical period for pain not strictly related to the surgical wound site being administered the study drug; significant medical conditions or laboratory results that indicated an increased vulnerability to the study drugs and/or procedures; and any clinically significant event or condition uncovered during the surgery that might have rendered the patient medically unstable or complicated the postsurgical course. Patients with a history of opioid or alcohol abuse/addiction were also excluded.

\section{Study design}

The milligram dose of liposome bupivacaine is expressed as the free base (ie, $266 \mathrm{mg}$ of bupivacaine base is chemically equivalent to $300 \mathrm{mg}$ of bupivacaine $\mathrm{HCl}$; Table 1 ). An overview of the 10 studies is shown in Table $2 .^{15-21}$ There were three studies that assessed the safety and efficacy of liposome bupivacaine in patients undergoing hemorrhoidectomy (studies 4, 7, and 9), two in patients undergoing inguinal hernia repair (studies 1 and 2), two in patients undergoing total knee arthroplasty (studies 3 and 6), two in patients undergoing breast augmentation (studies 5 and 8), and one in patients undergoing bunionectomy (study 10). Studies 1 and 5 included commercially available bupivacaine $\mathrm{HCl}$ without epinephrine (Marcain ${ }^{\circledR}$ Polyamp Steripack, 0.5\%; AstraZeneca UK Limited, Bedfordshire, UK) as an active comparator; studies 2, 3, 4, 6, 7, and 8 included bupivacaine
Table I Milligram dose equivalents for liposome bupivacaine expressed as the free base and bupivacaine $\mathrm{HCl}$

\begin{tabular}{ll}
\hline $\begin{array}{l}\text { Dose of liposome bupivacaine } \\
\text { expressed as the free base }(\mathbf{m g})\end{array}$ & $\begin{array}{l}\text { Equivalent dose of } \\
\text { bupivacaine } \mathbf{H C l}(\mathbf{m g})\end{array}$ \\
\hline 66 & 75 \\
93 & 105 \\
106 & 120 \\
133 & 150 \\
155 & 175 \\
160 & 180 \\
199 & 225 \\
266 & 300 \\
306 & 345 \\
310 & 350 \\
399 & 450 \\
532 & 600 \\
\hline
\end{tabular}

Abbreviation: $\mathrm{HCl}$, hydrochloride.

$\mathrm{HCl}$ with epinephrine (Marcaine ${ }^{\circledR} 0.5 \%$ with epinephrine 1:200,000; Hospira, Inc., Lake Forest, IL); and studies 9 and 10 included a placebo control arm ( $0.9 \%$ sodium chloride). Dose levels of liposome bupivacaine ranged from 66 to $532 \mathrm{mg}$ across studies. In each study, a single dose of study drug was administered intraoperatively via local administration at the end of surgery (day 1), immediately prior to wound closure. When called for by the relevant study protocol, patients received other analgesic medications for postsurgical pain as part of a multimodal pain management strategy.

\section{Efficacy assessments}

Pain intensity after surgery was measured using an 11-point numeric rating scale $(\mathrm{NRS})$ where $0=$ no pain and $10=$ worst possible pain. The NRS was used in all studies except study 1 , in which a $100 \mathrm{~mm}$ length visual analog scale $(0=$ no pain and $100=$ most severe pain possible) was used to measure pain intensity. Pain intensity scores were collected through at least 72 hours after study drug administration. Cumulative pain score as reflected in the area under the curve (AUC) of NRS scores through the last timed assessment, and through other time points, were derived for each study. Comparisons of mean AUC of NRS scores assessed through 24 and 72 hours for each study (except study 5; breast augmentation) while patients were at rest are presented in this analysis. Study 5 was not included in the analysis of AUC of NRS scores because patients were given liposome bupivacaine in one breast pocket and bupivacaine $\mathrm{HCl}$ in the other breast pocket; the majority of times that an opioid was taken it could not be attributed to pain in either the breast treated with liposome bupivacaine or the breast treated with bupivacaine $\mathrm{HCl}$.

Other key efficacy measures included time to first postsurgical use of opioid rescue medication, proportion 


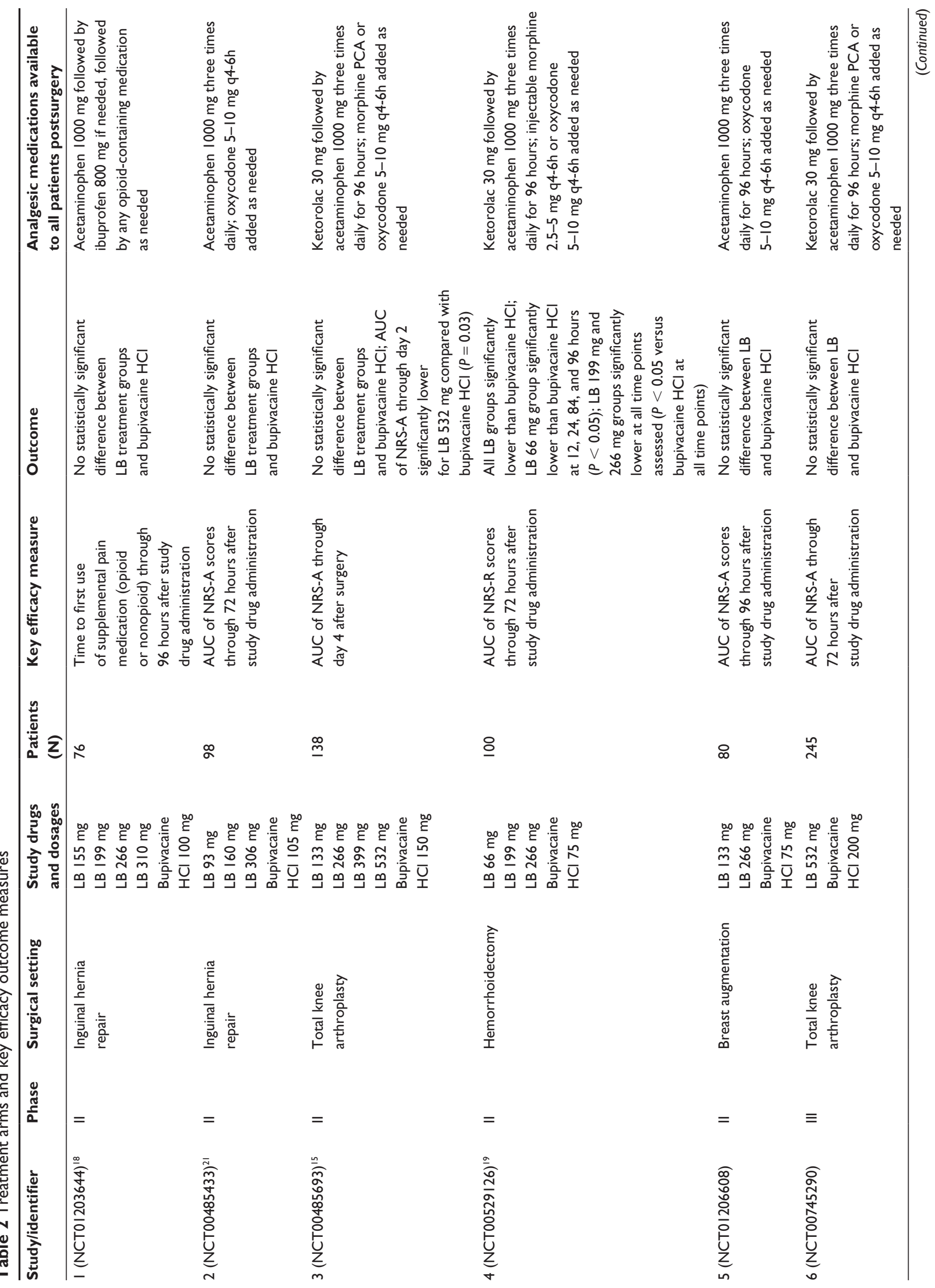




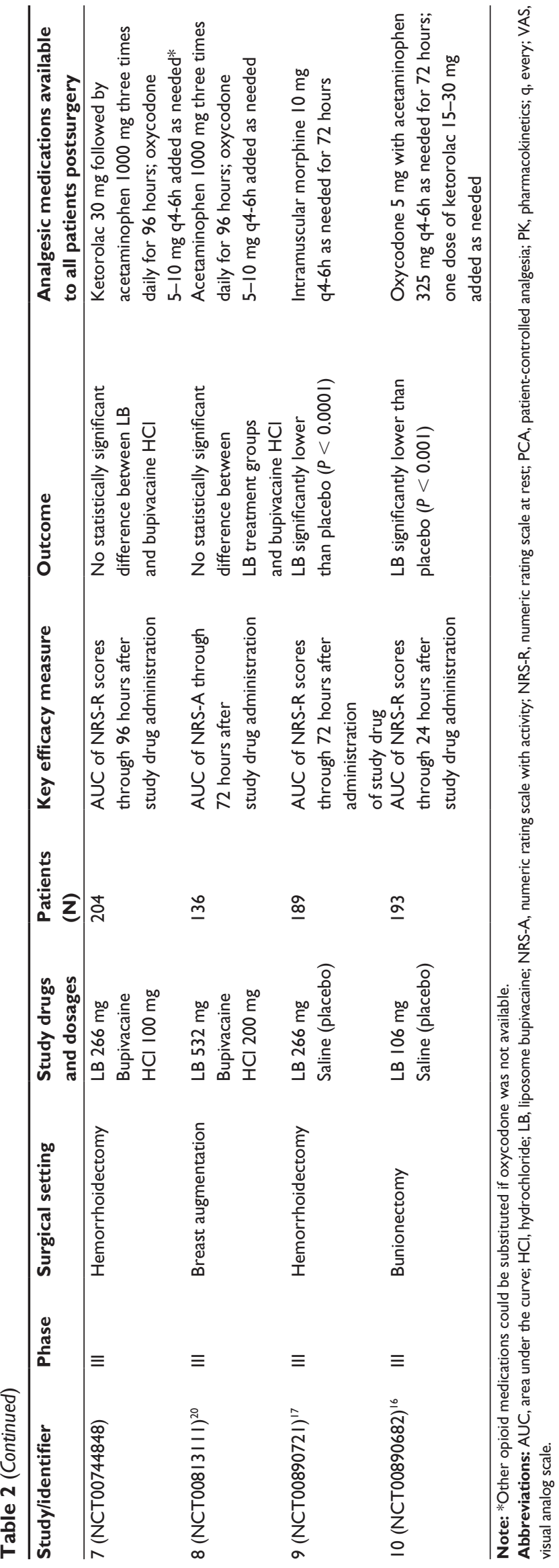

of patients who received no supplemental opioid rescue medication, total amount ( $\mathrm{mg}$ ) of opioid rescue medication consumed, and patient and caregiver ratings of satisfaction with postsurgical analgesia. Patient satisfaction was measured using a four-point categorical scale (poor to very good $)$ in study 1 , an 11-point numeric scale $(0=$ completely unsatisfied with analgesia; $10=$ completely satisfied with analgesia) in studies 6 and 7, and a five-point categorical scale (extremely dissatisfied to extremely satisfied) in studies 8, 9, and 10. Patient satisfaction ratings were not assessed in studies 2, 3, 4, and 5. Blinded care provider satisfaction with postsurgical analgesia was measured using a fourpoint categorical scale (poor to very good) in study 1 and an 11-point numeric scale ( $0=$ completely unsatisfied with analgesia; $10=$ completely satisfied with analgesia) in studies $2,3,4,5$, and 7 . This assessment was not conducted in studies $6,8,9$, and 10 .

\section{Data analyses}

The calculation of cumulative pain intensity scores (AUC of NRS) for each treatment arm in studies 8, 9, and 10 incorporated the use of rescue pain medications by imputing the worst pain intensity score prior to the use of a pain medication and carrying that value forward for a specified time period based on the half-life of the rescue pain medication. This imputation method is referred to as the "windowed worst observation carried forward + last observation carried forward" (wWOCF + LOCF). This method was retrospectively applied for analysis of AUC of NRS data from the other studies, with the exception of studies 3 and 6 , in which patients used a patient-controlled analgesia pump and study 5 in which patients received liposome bupivacaine in one breast pocket and bupivacaine $\mathrm{HCl}$ in the other breast pocket. In studies 3 and 6, the time and amount of rescue medication administered was not recorded each time the patient-controlled analgesia pump was used. In the wWOCF + LOCF analyses, missing scores were replaced in one of three ways: by the median score from other patients at the same time point in the same treatment group if before the first nonmissing score; by LOCF if after the last nonmissing score; and by linear interpolation if between two nonmissing scores. The intent-to-treat population included all randomized patients who received the study drug and was based on the treatment group to which patients were randomized. The intent-to-treat population was used for analyses of AUC of NRS via the wWOCF + LOCF imputation method. Analysis of other efficacy measures in each study was conducted using the full analysis population, which included all patients who 
Table 3 Patient disposition (pooled intent-to-treat population)

\begin{tabular}{|c|c|c|c|c|c|}
\hline & \multicolumn{3}{|c|}{ Liposome bupivacaine } & \multirow{2}{*}{$\begin{array}{l}\text { Bupivacaine } \mathrm{HCl} \\
(\mathrm{n}=446)\end{array}$} & \multirow{2}{*}{$\begin{array}{l}\text { Placebo } \\
(n=190)\end{array}$} \\
\hline & $\begin{array}{l}\leq 266 \mathrm{mg} \\
(\mathrm{n}=545)\end{array}$ & $\begin{array}{l}>266 \mathrm{mg} \\
(\mathrm{n}=278)\end{array}$ & $\begin{array}{l}\text { All doses } \\
(n=823)\end{array}$ & & \\
\hline Patients who terminated early, n (\%) & $9(1.7)$ & $35(12.6)$ & $44(5.3)$ & $49(11.0)$ & $6(3.2)$ \\
\hline \multicolumn{6}{|l|}{ Reason for early termination, $\mathrm{n}(\%)$} \\
\hline Death & 0 & 0 & 0 & $\mathrm{I}(0.2)$ & 0 \\
\hline Adverse event & 0 & I $(0.4)$ & $I(0.1)$ & $2(0.4)$ & $\mathrm{I}(0.5)$ \\
\hline Lost to follow-up & $2(0.4)$ & $3(1.1)$ & $5(0.6)$ & $12(2.7)$ & 0 \\
\hline Patient withdrew & $3(0.6)$ & $3(1.1)$ & $6(0.7)$ & $6(1.3)$ & $5(2.6)$ \\
\hline Other & $4(0.7)$ & $26(9.4)$ & $30(3.6)$ & $27(6.1)$ & 0 \\
\hline Not reported & 0 & $2(0.7)$ & $2(0.2)$ & $\mathrm{I}(0.2)$ & 0 \\
\hline
\end{tabular}

Abbreviation: $\mathrm{HCl}$, hydrochloride.

received the study drug, underwent the surgical procedure, and had sufficient data to calculate a cumulative pain score (AUC of NRS). The safety population included all patients who received at least one dose of study drug and was based on treatment actually received. For total postoperative consumption of opioid rescue medication, all opioids were converted to an equianalgesic parenteral morphine amount using standard conversion factors. ${ }^{22}$

Comparisons of liposome bupivacaine with bupivacaine $\mathrm{HCl}$ or placebo for efficacy measures were made using analysis of variance, Cochran-Mantel-Haenszel tests, or logrank tests as appropriate. All statistical tests were performed against a two-sided alternative hypothesis with a significance level of $5 \%(\alpha=0.05)$, and all confidence intervals calculated were two-sided $95 \%$ confidence intervals.

\section{Results}

Liposome bupivacaine was administered to a total of 823 patients across the 10 studies at doses ranging from $66 \mathrm{mg}$ to $532 \mathrm{mg}$. Results from key efficacy measures in each of the 10 studies are summarized in Table $2 .{ }^{15-21}$ Patient disposition is summarized in Table 3 and patient demographics and other baseline characteristics are shown in Table 4 . Since there was a $\leq 1 \%$ difference between the total number of patients for each study treatment in the intent-to-treat and safety populations, the demographics and baseline characteristics were expected to be similar in both populations.

\section{Cumulative pain scores}

Between-group differences in cumulative pain scores (AUC of NRS) through 24 and 72 hours across studies are shown in

Table 4 Patient demographics and other baseline characteristics (pooled safety population)

\begin{tabular}{|c|c|c|c|c|c|}
\hline & \multicolumn{3}{|c|}{ Liposome bupivacaine } & \multirow{2}{*}{$\begin{array}{l}\text { Bupivacaine } \mathrm{HCl}^{*} \\
(\mathrm{n}=446)\end{array}$} & \multirow{2}{*}{$\begin{array}{l}\text { Placebo } \\
(n=190)\end{array}$} \\
\hline & $\begin{array}{l}\leq 266 \mathrm{mg} \\
(\mathrm{n}=545)\end{array}$ & $\begin{array}{l}>266 \mathrm{mg} \\
(\mathrm{n}=278)\end{array}$ & $\begin{array}{l}\text { All doses } \\
(n=823)\end{array}$ & & \\
\hline Age, years, mean (SD) & $47.6(14.2)$ & $55.9(17.6)$ & $50.4(15.9)$ & $49.5(16.7)$ & $45.9(12.9)$ \\
\hline \multicolumn{6}{|c|}{ Age category, years, n (\%) } \\
\hline$<40$ & $168(30.8)$ & $70(25.2)$ & $238(28.9)$ & $144(32.3)$ & $63(33.2)$ \\
\hline $40-64$ & $313(57.4)$ & $99(35.6)$ & $412(50.1)$ & $210(47.1)$ & $112(58.9)$ \\
\hline$\geq 65$ & 64 (II.7) & $107(38.5)$ & I7| (20.8) & $92(20.6)$ & I5. (7.9) \\
\hline Not reported & 0 & $2(0.7)$ & $2(0.2)$ & 0 & 0 \\
\hline \multicolumn{6}{|l|}{ Sex, n (\%) } \\
\hline Male & $304(55.8)$ & $94(33.8)$ & $398(48.4)$ & $179(40.1)$ & $79(4 \mid .6)$ \\
\hline Female & $24 I(44.2)$ & $184(66.2)$ & $425(5 \mathrm{I} .6)$ & $267(59.9)$ & III (58.4) \\
\hline \multicolumn{6}{|l|}{ Race, n (\%) } \\
\hline Caucasian & $469(86.1)$ & $240(86.3)$ & $709(86.1)$ & $384(86.1)$ & $166(87.4)$ \\
\hline Non-Caucasian & $76(13.9)$ & $36(12.9)$ & $112(13.6)$ & $62(13.9)$ & $24(12.6)$ \\
\hline Not reported & 0 & $2(0.7)$ & $2(0.2)$ & 0 & 0 \\
\hline \multicolumn{6}{|l|}{ ASA class, n (\%) } \\
\hline $\mathrm{I}-2$ & $477(87.5)$ & $182(65.5)$ & $659(80.1)$ & $354(79.4)$ & $187(98.4)$ \\
\hline $3-4$ & $51(9.4)$ & $84(30.2)$ & $135(16.4)$ & $82(18.4)$ & $3(1.6)$ \\
\hline Not reported & $17(3.1)$ & $12(4.3)$ & $29(3.5)$ & $10(2.2)$ & 0 \\
\hline
\end{tabular}

Notes: *Bupivacaine $\mathrm{HCl}$ doses of $75 \mathrm{mg}$ to $200 \mathrm{mg}$ were used across studies. Bupivacaine $\mathrm{HCl}$ with epinephrine $1: 200,000$ was used in studies 2, 3, 4, 6, 7, 8, 9, and I0. Bupivacaine $\mathrm{HCl}$ without epinephrine was used in studies I and 5 .

Abbreviations: ASA, American Society of Anesthesiologists; $\mathrm{HCl}$, hydrochloride; SD, standard deviation. 
Figures 1 and 2, respectively. In general, the higher doses of liposome bupivacaine were associated with lower cumulative pain scores compared with placebo and bupivacaine $\mathrm{HCl}$. In the cumulative pain (AUC of NRS) analysis, there were 17 treatment arms comparing liposome bupivacaine with bupivacaine $\mathrm{HCl}$ in active control studies. Between-group differences were statistically significant in favor of liposome bupivacaine in six of these treatment arms through 24 hours and in five treatment arms through 72 hours $(P<0.05)$.

\section{Time to first postsurgical use of opioid rescue medication}

The time to first postsurgical use of supplemental opioid pain medication was pooled across studies (except study 5) for all liposome bupivacaine doses combined, for bupivacaine $\mathrm{HCl}$, and for placebo (Table 5). The median time to first postsurgical use of rescue opioid medication was significantly longer with liposome bupivacaine ( 9.3 hours) compared with bupivacaine $\mathrm{HCl}(6.4$ hours; $P=0.013)$ and placebo (3.6 hours; $P<0.0001$ ).

\section{Proportion of patients avoiding use of opioid rescue medication}

In study 9 (hemorrhoidectomy), the proportion of patients avoiding rescue medication through 72 hours after surgery was significantly lower in favor of liposome bupivacaine $266 \mathrm{mg}$ ( $28 \%$ avoided use of rescue opioids) compared with placebo (10\% avoided use of rescue opioids; $P=0.0007$ ). Between-group differences did not reach statistical significance in the other placebo-controlled Phase III study (study 10), or the seven studies that included comparisons of liposome bupivacaine with bupivacaine $\mathrm{HCl}$. Study 5

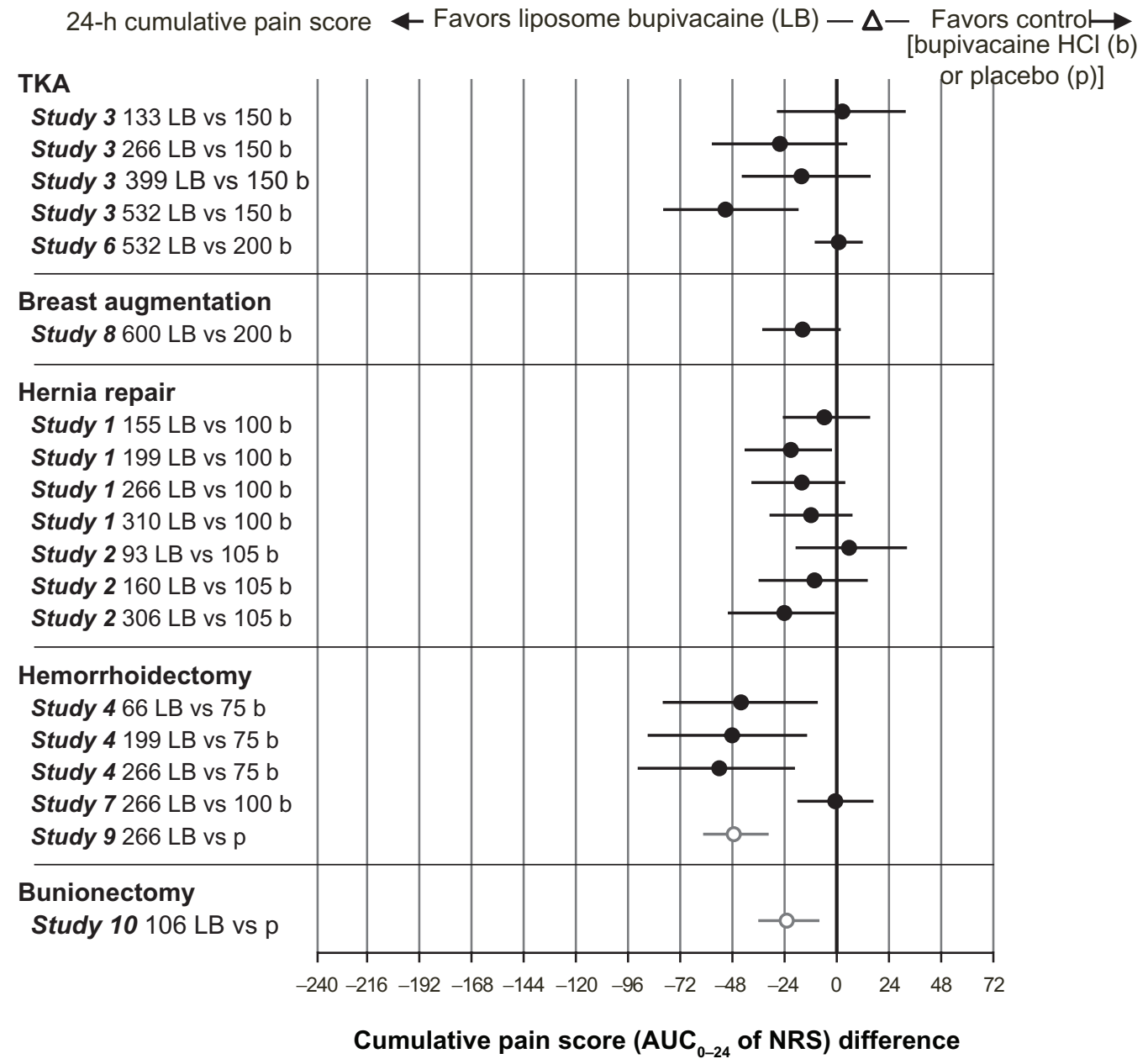

Figure I Cumulative pain score (AUC ${ }_{0-24}$ of NRS).

Notes: Differences in AUC for pain at rest from 0 to 24 hours between liposome bupivacaine and control groups. Circles represent the difference in means, and bars represent the $95 \%$ confidence intervals for the difference in means. The perpendicular zero line indicates no difference between liposome bupivacaine and controls. If a confidence interval does not cross the zero line, there is a statistically significant difference $(P<0.05)$ between liposome bupivacaine and controls.

Abbreviations: AUC, area under the curve; $\mathrm{HCl}$, hydrochloride; NRS, numeric rating scale; TKA, total knee arthroplasty. 


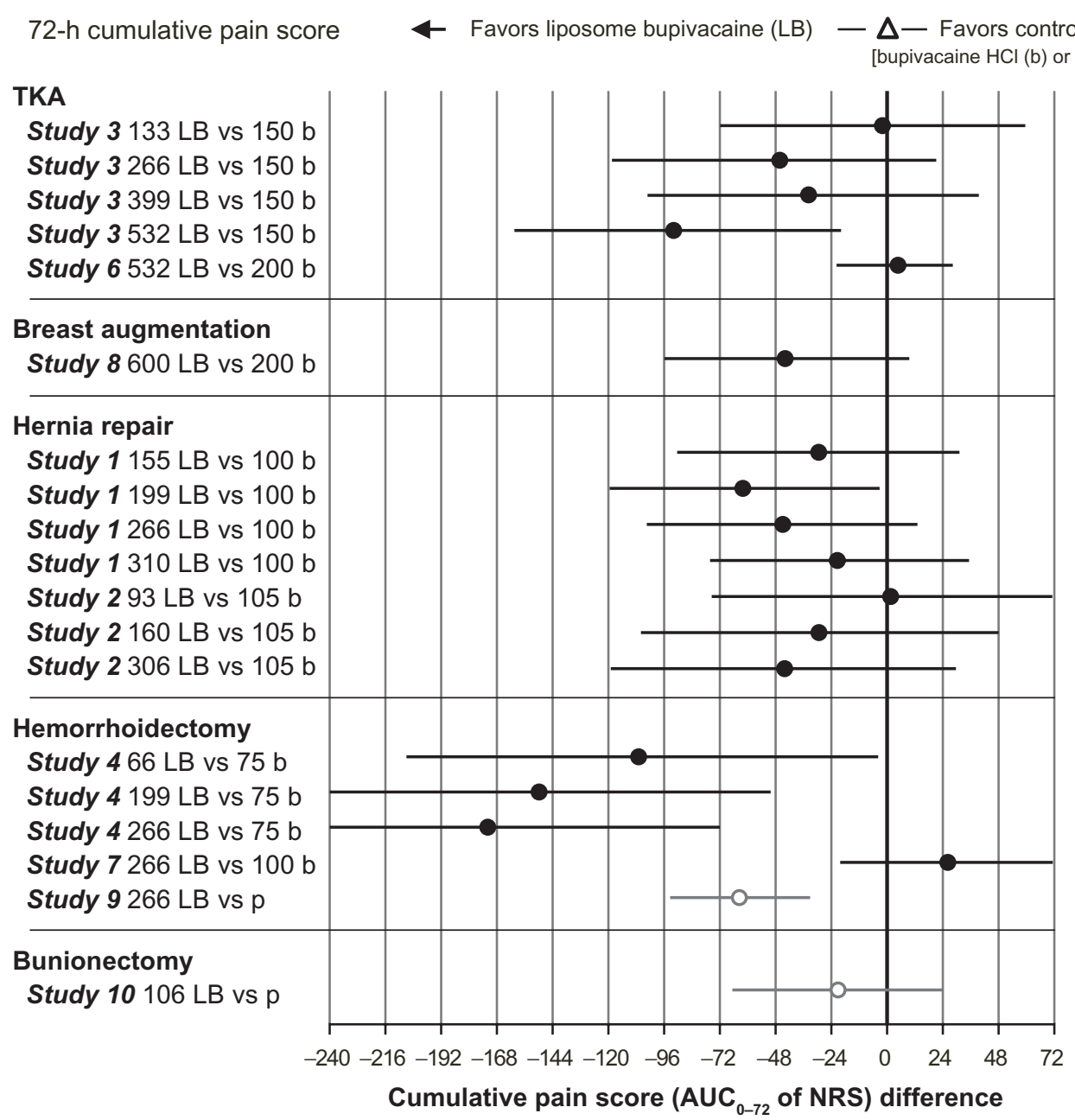

Figure 2 Cumulative pain score (AUC $\mathrm{C}_{0-72}$ of NRS).

Notes: Differences in AUC for pain at rest from 0 to 72 hours between liposome bupivacaine and control groups. Circles represent the difference in means and bars represent the $95 \%$ confidence intervals for the difference in means. The perpendicular zero line indicates no difference between liposome bupivacaine and controls. If a confidence interval does not cross the zero line, there is a statistically significant difference $(P<0.05)$ between liposome bupivacaine and controls.

Abbreviations: AUC, area under the curve; $\mathrm{HCl}$, hydrochloride; NRS, numeric rating scale; TKA, total knee arthroplasty.

did not include a statistical comparison between liposome bupivacaine and bupivacaine $\mathrm{HCl}$ for this efficacy measure because patients received liposome bupivacaine in one breast and bupivacaine $\mathrm{HCl}$ in the other breast.

\section{Total postsurgical consumption of opioid rescue medication}

Total postsurgical consumption of opioid rescue medication was statistically significantly lower for liposome bupivacaine-treated patients than for the comparator in four studies (two active-controlled, two placebo-controlled) at 24 hours postsurgery, and in two studies (one activecontrolled, one placebo-controlled) at 72 hours postsurgery (Table 6). The higher doses of liposome bupivacaine (266 mg and $532 \mathrm{mg}$ ) were more frequently associated with a significant reduction in total postsurgical consumption of opioids than lower doses. There were no statistically significant differences observed between the liposome bupivacaine treatment arms and bupivacaine $\mathrm{HCl}$ in the other studies.

\section{Patient and blinded care provider satisfaction with postoperative analgesia}

In the studies that included assessment of patient ratings of postsurgical analgesia (studies 1, 6, 7, 8, 9, and 10), the liposome bupivacaine group in study 9 showed statistically significantly better patient satisfaction scores than the comparator. At 24 hours after surgery in this study, $95 \%$ of patients in the liposome bupivacaine 266 mg group were "satisfied" or "extremely satisfied" with their postoperative analgesia compared with $72 \%$ in the placebo group $(P=0.0007)$. At 72 hours after surgery, percentages were $95 \%$ and $73 \%$, respectively $(P=0.0007)$. 
Table $\mathbf{5}$ Time to first postsurgical use of supplemental opioid pain medication through 72 hours (pooled intent-to-treat population)

\begin{tabular}{llll}
\hline & $\begin{array}{l}\text { Liposome } \\
\text { bupivacaine } \\
(\mathbf{n}=\mathbf{7 8 0})\end{array}$ & $\begin{array}{l}\text { Bupivacaine } \\
\mathbf{H C l} \\
(\mathbf{n}=\mathbf{4 0 9})\end{array}$ & $\begin{array}{l}\text { Placebo } \\
(\mathbf{n}=1 \mathbf{9 0})\end{array}$ \\
\hline $\begin{array}{l}\text { Number of patients } \\
\text { who used supplemental }\end{array}$ & 619 & 343 & 180 \\
$\begin{array}{l}\text { medication } \\
\text { Quartiles* (hours) }\end{array}$ & & & \\
$\quad \begin{array}{l}\text { First quartile } \\
\text { Median (95\% Cl) }\end{array}$ & 1.8 & 0.7 & 1.2 \\
Third quartile & 31.8 & 25.3 & 5.4 \\
\hline
\end{tabular}

Notes: *First quartile, $25 \%$ started using pain medication; median, $50 \%$ started using pain medication; third quartile, $75 \%$ started using pain medication; ${ }^{\dagger} P=0.013$ versus bupivacaine $\mathrm{HCl}$ and $P<0.0001$ versus placebo.

Abbreviations: $\mathrm{Cl}$, confidence interval; $\mathrm{HCl}$, hydrochloride.

The other studies showed no statistically significant betweengroup differences for this assessment.

For assessments of care provider satisfaction with postoperative analgesia (studies 1, 2, 3, 4, 5, and 7), statistically significant differences were observed between the liposome bupivacaine and bupivacaine $\mathrm{HCl}$ groups in study 1 at 24 hours, where $100 \%$ of care providers of patients in the liposome bupivacaine $199 \mathrm{mg}$ group rated satisfaction as "good" or "very good" on a categorical scale versus $81 \%$ in the bupivacaine $\mathrm{HCl} 100 \mathrm{mg}$ group (95\% confidence interval for difference in percentages, 4.1-34.4). Statistically significant differences in favor of liposome bupivacaine were also observed in study 3 , where mean satisfaction ratings, based on an 11-point NRS $(0=$ completely unsatisfied; $10=$ completely satisfied), were 9.2 in the liposome bupivacaine $532 \mathrm{mg}$ group and 8.3 in the bupivacaine $\mathrm{HCl} 150 \mathrm{mg}$ group $(P=0.045)$ at day 8 , and in study 4 where mean ratings in the liposome bupivacaine $266 \mathrm{mg}$ and bupivacaine $\mathrm{HCl} 75 \mathrm{mg}$ groups were 7.4 and 6.0 , respectively $(P=0.03)$ at 96 hours. No statistically significant between-group differences were observed for this assessment in the other studies.

Liposome bupivacaine was well tolerated across the 823 patient exposures in these 10 studies, and the adverse event profile was similar for liposome bupivacaine and bupivacaine $\mathrm{HCl}$. Overall, 62\% (508 of 823) of patients treated with liposome bupivacaine reported at least one adverse event compared with $75 \%$ (334 of 446) for bupivacaine $\mathrm{HCl}$ and $43 \%$ (82 of 190) for placebo. The incidence of adverse events generally increased with increasing doses of either liposome bupivacaine or bupivacaine $\mathrm{HCl}$. Nausea, constipation, and vomiting were the most frequently reported adverse events in patients who received liposome bupivacaine or bupivacaine $\mathrm{HCl}$; these adverse events are frequently reported in patients receiving opioid medications.

\section{Discussion}

The highly subjective nature of pain perception and measurement is an inherent limitation of all pain studies. To mitigate this limitation, clinical studies conducted in the pain setting are typically designed to involve multiple metrics for measurement of treatment-related efficacy. Most wound

Table 6 Total postsurgical consumption of opioid rescue medication: studies with statistical differences between treatment groups

\begin{tabular}{|c|c|c|c|c|c|c|c|}
\hline \multicolumn{8}{|c|}{ Study $4^{19}$} \\
\hline \multicolumn{4}{|c|}{ Adjusted geometric mean total consumption through 24 hours (mg) } & \multicolumn{4}{|c|}{ Adjusted geometric mean total consumption through 72 hours (mg) } \\
\hline LB 66 & LB 199 & LB 266 & B 75 & LB 66 & LB 199 & LB 266 & B 75 \\
\hline$(n=25)$ & $(n=25)$ & $(n=25)$ & $(n=25)$ & $(n=25)$ & $(n=25)$ & $(n=25)$ & $(n=25)$ \\
\hline 8.0 & 7.2 & $4.2^{*}$ & 8.9 & 15.0 & 10.0 & $6.2^{\dagger}$ & 18.4 \\
\hline \multicolumn{8}{|c|}{ Study $8^{20}$} \\
\hline \multicolumn{4}{|c|}{ Mean total consumption through 24 hours (mg) } & \multicolumn{4}{|c|}{ Mean total consumption through 72 hours (mg) } \\
\hline LB 532 & B 200 & & & LB 532 & B 200 & & \\
\hline$(n=60)$ & $(n=62)$ & & & $(n=60)$ & $(n=62)$ & & \\
\hline $6.1 *$ & 9.3 & & & 13.5 & 20.4 & & \\
\hline \multicolumn{8}{|c|}{ Study $9^{17}$} \\
\hline \multicolumn{4}{|c|}{ Adjusted geometric mean total consumption through 24 hours (mg) } & \multicolumn{4}{|c|}{ Adjusted geometric mean total consumption through 72 hours (mg) } \\
\hline LB 266 & Placebo & & & LB 266 & Placebo & & \\
\hline$(n=94)$ & $(n=93)$ & & & $(n=94)$ & $(n=93)$ & & \\
\hline $5.4^{\ddagger}$ & 12.9 & & & $9.9^{\dagger}$ & 18.2 & & \\
\hline \multicolumn{8}{|c|}{ Study $10^{16}$} \\
\hline \multicolumn{4}{|c|}{ Adjusted geometric mean total consumption through 24 hours (mg) } & \multicolumn{4}{|c|}{ Adjusted geometric mean total consumption through 72 hours (mg) } \\
\hline LB 106 & Placebo & & & LB 106 & Placebo & & \\
\hline$(n=97)$ & $(n=96)$ & & & $(n=97)$ & $(n=96)$ & & \\
\hline $3.8^{\dagger}$ & 4.7 & & & 11.3 & II.I & & \\
\hline
\end{tabular}

Notes: ${ }^{* P}<0.05 ;{ }^{\dagger} P<0.01$; ${ }^{\ddagger} P<0.0001$.

Abbreviations: B, bupivacaine $\mathrm{HCl}$ (hydrochloride); LB, liposome bupivacaine. 
infiltration studies in the liposome bupivacaine development program included an assessment of cumulative pain over time as the primary efficacy measure, while secondary measures focused on outcomes such as pain intensity scores and opioid usage at specific time points. Pooling of the efficacy data allowed for a more detailed analysis of outcome measures that were utilized across studies. In this pooled analysis of efficacy, results from 823 patients who received single-dose, locally administered liposome bupivacaine across 10 studies in five different surgical models, liposome bupivacaine was shown to provide prolonged analgesia for up to 72 hours after surgery. Across seven active-controlled studies, there were 17 treatment arms comparing liposome bupivacaine and bupivacaine $\mathrm{HCl}$. Between-group differences in cumulative pain scores trended in favor of liposome bupivacaine through 72 hours postsurgery in 14 of the 17 treatment arms, reaching statistical significance $(P<0.05)$ in five treatment arms. Cumulative pain scores trended in favor of bupivacaine $\mathrm{HCl}$ in three of the 17 treatment arms, none of which reached statistical significance at any timed assessment.

The cumulative pain score results were supported by the results of other efficacy measures. The median time to first postsurgical use of supplemental opioid pain medication was 3 hours later with liposome bupivacaine versus bupivacaine $\mathrm{HCl}(P=0.013)$ and 6 hours later than placebo $(P<0.0001)$. Between-group differences in proportion of patients who avoided use of rescue opioids and total consumption of rescue opioids after surgery also trended in favor of liposome bupivacaine in most of the studies analyzed. The greatest reductions in opioid use were observed in the treatment arms that received the highest doses of liposome bupivacaine (266 mg or $532 \mathrm{mg}$ ). Also, the between-group comparisons showed the higher dose levels of liposome bupivacaine were more frequently associated with higher patient and caregiver satisfaction scores than lower dose levels. Local infiltration of liposome bupivacaine resulted in significant systemic plasma levels of bupivacaine, which could persist for 96 hours; systemic plasma levels of bupivacaine following administration of liposome bupivacaine were not correlated with local efficacy.

A limitation of this post hoc analysis is that the results cannot be extrapolated to surgical models not examined in the 10 studies pooled for this analysis or to patient populations receiving liposome bupivacaine via administration routes other than wound infiltration. Pooling of efficacy results was not prespecified in protocols for the individual studies.

\section{Conclusion}

In conclusion, treatment with liposome bupivacaine resulted in lower pain scores and reduced opioid consumption during the first 72 hours after surgery in several surgical models. A reduction in postsurgical pain may result in less need for supplemental opioid pain medications, fewer opioid-related adverse events, and a better recovery experience for patients, which may offer an economic benefit to health care systems. Based on this retrospective integrated analysis of multiple efficacy measures, liposome bupivacaine appears to be a potentially useful therapeutic option for prolonged reduction of postsurgical pain in soft tissue and orthopedic surgeries.

\section{Acknowledgment}

The authors acknowledge Barbara Elashoff for her assistance with the statistical analysis.

\section{Disclosure}

Editorial assistance was provided by Peloton Advantage, LLC, and supported by Pacira Pharmaceuticals, Inc. SDB has received research support from Pacira Pharmaceuticals, Inc. GP is an employee of Pacira Pharmaceuticals, Inc. SR, $\mathrm{KB}, \mathrm{SRG}$, and KAC report no conflicts of interest in this work.

\section{References}

1. Apfelbaum JL, Chen C, Mehta SS, Gan TJ. Postoperative pain experience: results from a national survey suggest postoperative pain continues to be undermanaged. Anesth Analg. 2003;97(2):534-540.

2. Warfield CA, Kahn CH. Acute pain management. Programs in US hospitals and experiences and attitudes among US adults. Anesthesiology. 1995;83(5):1090-1094.

3. Beauregard L, Pomp A, Choiniere M. Severity and impact of pain after day-surgery. Can J Anaesth. 1998;45(4):304-311.

4. Practice guidelines for acute pain management in the perioperative setting: an updated report by the American Society of Anesthesiologists Task Force on Acute Pain Management. Anesthesiology. 2012;116(2): 248-273.

5. White PF. Multimodal analgesia: its role in preventing postoperative pain. Curr Opin Investig Drugs. 2008;9(1):76-82.

6. Jirasiritham S, Tantivitayatan K, Jirasiritham S. Perianal blockage with $0.5 \%$ bupivacaine for postoperative pain relief in hemorrhoidectomy. J Med Assoc Thai. 2004;87(6):660-664.

7. Chester JF, Stanford BJ, Gazet JC. Analgesic benefit of locally injected bupivacaine after hemorrhoidectomy. Dis Colon Rectum. 1990; 33(6):487-489.

8. Moiniche S, Kehlet H, Dahl JB. A qualitative and quantitative systematic review of preemptive analgesia for postoperative pain relief: the role of timing of analgesia. Anesthesiology. 2002;96(3):725-741.

9. Moiniche S, Mikkelsen S, Wetterslev J, Dahl JB. A qualitative systematic review of incisional local anaesthesia for postoperative pain relief after abdominal operations. Br J Anaesth. 1998;81(3): 377-383.

10. Yoost TR, McIntyre M, Savage SJ. Continuous infusion of local anesthetic decreases narcotic use and length of hospitalization after laparoscopic renal surgery. J Endourol. 2009;23(4):623-626. 
11. Mahabir RC, Peterson BD, Williamson JS, Valnicek SM, Williamson DG, East WE. Locally administered ketorolac and bupivacaine for control of postoperative pain in breast augmentation patients. Plast Reconstr Surg. 2004;114(7):1910-1916.

12. Wheatley GH III, Rosenbaum DH, Paul MC, et al. Improved pain management outcomes with continuous infusion of a local anesthetic after thoracotomy. J Thorac Cardiovasc Surg. 2005;130(2): 464-468.

13. International Conference on Harmonisation Working Group. ICH Harmonised Tripartite Guideline: Guideline for Good Clinical Practice E6 (R1). International Conference on Harmonisation of Technical Requirements for Registration of Pharmaceuticals for Human Use; June 10, 1996; Washington, DC. Available at: http:/www.ich.org/fileadmin/ Public_Web_Site/ICH_Products/Guidelines/Efficacy/E6_R1/Step4/ E6_R1_Guideline.pdf. Accessed March 1, 2011.

14. World Medical Association. Declaration of Helsinki: ethical principles for medical research involving human subjects. Available at: http://www. wma.net/en/30publications/10policies/b3/17c.pdf. Accessed April 26, 2010.

15. Bramlett KW, Jones RK, Pink M, Pink T. A single administration of DepoBupivacaine intraoperatively provides analgesia and reduction in use of rescue opiates compared to bupivacaine $\mathrm{HCl}$ in patients undergoing total knee arthroplasty [Abstract 0278]. Presented at the XXXVI Biennial World Congress of the International College of Surgeons, December 3-6, 2008, Vienna, Austria.

16. Golf M, Daniels SE, Onel E. A phase 3, randomized, placebo-controlled trial of DepoFoam $(\mathrm{R})$ bupivacaine (extended-release bupivacaine local analgesic) in bunionectomy. Adv Ther. 2011;28(9):776-788.
17. Gorfine S, Krivokapic Z. Extended-release multivesicular liposome bupivacaine (Exparel ${ }^{\mathrm{TM}}$ ) is superior to placebo for posthemorrhoidectomy pain reduction [Abstract P230]. Dis Colon Rectum. 2011; 54(5): 174

18. Langford RM, Chappell GM, Karrasch JA. A single administration of DepoBupivacaine intraoperatively results in prolonged detectable plasma bupivacaine and analgesia in patients undergoing inguinal hernia repair [Abstract P-9088]. Presented at the Annual Postgraduate Assembly in Anesthesiology of the New York State Society of Anesthesiologists, December 12-16, 2008, New York, NY.

19. Miller H, Terem TM, Kheladze K, Mosidze B. A single administration of DepoBupivacaine intraoperatively provides three-day analgesia and reduction in use of rescue opioids in patients undergoing hemorrhoidectomy [Abstract]. Presented at the Annual Meeting of the American Society of Colon and Rectal Surgeons, May 2-6, 2009, Hollywood, FL.

20. Smoot JD, Bergese SD, Onel E, Williams HT, Hedden W. The efficacy and safety of DepoFoam ${ }^{\circledR}$ bupivacaine in patients undergoing bilateral, cosmetic, submuscular augmentation mammoplasty: a randomized, double-blind, active-control study. Aesthet Surg J. 2012;32(1):69-76.

21. White PF, Schooley GL, Ardeleanu M. Analgesia following a single administration of depobupivacaine intraoperatively in patients undergoing inguinal herniorrhaphy: preliminary dose-ranging studies [Abstract S-242]. Anesth Analg. 2009;108 Suppl 3S:S242.

22. Reisine T, Pasternak G. Opioid analgesics and antagonists. In: Hardman JG, Limbard LE, Molinoff PB, editors. Goodman and Gilman's The Pharmacological Basis of Therapeutics. 9th ed. New York, NY: McGraw-Hill; 1996.
Journal of Pain Research

\section{Publish your work in this journal}

The Journal of Pain Research is an international, peer-reviewed, open access, online journal that welcomes laboratory and clinical findings in the fields of pain research and the prevention and management of pain. Original research, reviews, symposium reports, hypothesis formation and commentaries are all considered for publication.

\section{Dovepress}

The manuscript management system is completely online and includes a very quick and fair peer-review system, which is all easy to use. Visit http://www.dovepress.com/testimonials.php to read real quotes from published authors. 